\title{
Developing an Image Processing-Based Method to Objectively Decide the Conspicuousness of Permanent Facial Scar
}

\author{
Ali Gelir ${ }^{2}$, Aysegul S. Yilmaz ${ }^{1}$, Faruk Asicioglu ${ }^{1 *}$, Omer F. Kadi, Kadir B. Yildirim², Mustafa Okudan ${ }^{3}$, Kagan Gurpinar $^{4}$ \\ ${ }^{1}$ Istanbul University-Cerrahpasa, Institute of Forensic Science and Legal Medicine \\ ${ }^{2}$ Istanbul Technical University, Physics Engineering Department \\ ${ }^{3}$ Igdir University, Faculty of Health Sciences \\ ${ }^{4}$ Maltepe University, Faculty of Medicine
}

\begin{abstract}
Permanent facial scars cause serious trauma because the person has to live with a scar which s/he cannot easily hide. Conspicuous scar tissue will have a negative impact on psychology depending on how much importance they give to their appearance. Because of this reason, it is evaluated as an aggravated form of punishment in Turkish Criminal Law.

In this study, image processing software was used for the quantitative analysis of facial scars. The scar photographs were taken, and the relative colour difference of the scar region was measured concerning the nearby healthy area. The scar's physical properties, such as the length and the surface area, were measured by processing the photographs in software.

An evaluation of 100 cases showed that the relative color difference rate of $15 \%$ is critical for deciding the conspicuousness of permanent facial scar. Above this rate, the facial scar is visible by the naked eye without affecting any factors such as the physicians' visual acuity, attention, and experience. Otherwise, the scar's length and the surface area must also be evaluated for a correct decision about the conspicuousness of a permanent facial scar.

Keywords: Facial scar, Forensic Medicine, Image Processing, Forensic Science, Legal Medicine

Int J Eth Trauma Victimology (2021). DOI: 10.18099/ijetv.v7i02.5
\end{abstract}

\section{INTRODUCTION}

$\mathrm{P}$ hysical injury or a wound has been defined as damage to any part of the body due to a deliberate or an accidental application of mechanical force or other traumatic agent causing serious bodily harm. ${ }^{1,2}$ Facial scars are considered aesthetic damage that causes unpleasant modifications in both the static or dynamic facial expression recognized by everyone and affect the image of the person. ${ }^{3}$

It is reported that people who have symmetrical faces are more attractive to the opposite sex than those with asymmetrical faces and the social interactions with both sexes are easier. ${ }^{4}$ It is also known that anxiety and depression caused by the trauma of the face, increase with the healing process delay. ${ }^{5}$ It has also been reported that the disfiguration based on facial trauma makes the person unhappy, socially isolated and stigmatized. ${ }^{4-6}$ The aesthetic concerns of these patients lead to multidisciplinary surgical intervention more than once in many cases. ${ }^{7}$ It has been reported that facial trauma victims have more somatoform symptoms, an increased tendency to substance abuse, and decreased joy of life. ${ }^{8}$

Each trauma, depending on the level, affects individuals painfully and destructs them psychologically. Therefore, Turkish law is seeking a criterion to assess the perpetrator's penalty according to the severity of the trauma. In addition, the law has been concerned with the significance of the trauma result without being directly related to its level. A trauma that leads to a permanent and conspicuous facial scar is also evaluated in this context, and the penalty given to the perpetrator is more aggravated than the bare form of crime. ${ }^{9}$
Corresponding Author: Faruk Asicioglu, Istanbul UniversityCerrahpasa, Institute of Forensic Science and Legal Medicine, e-mail: faruk.asicioglu@iuc.edu.tr

How to cite this article: Gelir A, Yilmaz AS, Asicioglu F, Kadi OF, Yildirim KB, Okudan M, Gurpinar K. Developing an Image ProcessingBased Method to Objectively Decide the Conspicuousness of PermanentFacial Scar. Int JEth Trauma Victimology. 2021;7(2):23-29.

Source of support: The author(s) disclosed receipt of the following financial support for the research: This work was supported by the Scientific and Technological Research Council of Turkey (TUBiTAK) [Grant No. 216S896]

Conflict of interest: None

Received: 11/09/2021;

Received in revised form: $15 / 11 / 2021$;

Accepted:23/11/2021; Published:20/01/2022

In a criminal assault case, if the act of the perpetrator causes a permanent and conspicuous scar on the face of the victim, according to the criminal law of several countries like Bangladesh, Italy, Turkey, the punishment will be heavier than a simple form of offence, and it may also cause indemnity claims. The decision of the conspicuousness and permanence of the facial scar is determined by medical doctors at least six months after the incident via the observation of the victim's face from one or two meters away by the naked eye under adequate light (preferably daylight). However, the decision can be affected by many factors such as the visual acuity, attention, mood, sense of aesthetics, and experience of the physicians.

In this study, the relative color changes of the scar region concerning the nearby healthy region were calculated. 
Furthermore, the surface area and the length of the scar were measured to facilitate the physician's final decision on the significance and permanence of the facial scar with objective parameters.

\section{Materials and Methods}

The victims of all cases were selected randomly whom criminal courts sent to the 2nd Specified Committee of Turkey's National Council of Forensic Medicine. This Council is the main organization in the country which works under the jurisdiction of the Ministry of Justice. The Council has subunits in all cities of the country. The Council consists of laboratories that provide analysis and different specialized committees that give expert opinions by evaluating the case as a whole with the participation of experts from different disciplines. There are eight specialized committees of the Council, which are named with numbers. The aforementioned 2nd Specified Committee gives an expert opinion about all kinds of trauma that contain permanent and significant facial scar evaluation. It consists of board members from 12 different medical and forensic disciplines such as forensic medicine specialists, plastic and reconstructive surgery, general surgery, ear, nose, and throat surgery, ophthalmology, psychiatry, orthopedics, radiology, etc.

The photographs of the victims were taken by using Canon 1300D cameras on a tripod. During photograph taking, different lighting conditions were used. A ring led illuminator with different colors mounted on the lens and a polarizing filter (B and B Digital CPL 58mm filter) in front of the lens were used for lighting.

\section{Image Processing Procedure}

Processing the images was performed by using the ImageJ software, a Java-based image processing program developed by the National Institute of Health of the USA. ${ }^{10}$

The photographs were directly used for processing, i.e., no filtering or further modifications were applied to the photographs for analysis. The procedure for the quantitative analysis of the photographs can be explained as follows:

- Determining the relative color change between the scar region and the nearby intact area on the face. To obtain this color difference in the greyscale, the plot profile tool of the ImageJ software was used. For this purpose, first, the image

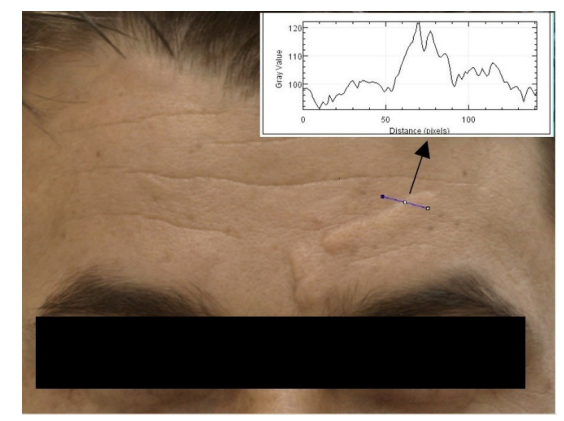

Figure 1: Sample photograph on which the colour profile was shown. A line was drawn through the healthy and the scar regions on the face, and a plot of the color values for this profile was given as a subfigure. is converted to an 8-bit grayscale image, and then a line that passes over the healthy part and the scar region was drawn to draw the colour profile plot through this line as seen in Figure 1. The relative colour change was calculated by taking the ratio of the averages of the healthy colour values and the scar regions on the face. The average colour values were calculated in the intervals in which the sharp changes, especially while passing from healthy to scar regions, were excluded. For example, in Figure 1, the interval 25 to 50 pixels and 65 to 85 pixels were used to calculate the average color values of the health and the scar regions, respectively.

- The second step of the analysis is selecting the scar region and then calculating the surface area of this region. The selection of the scar region is performed by using the Versatile Wand Tool ${ }^{11}$ plugin of the ImageJ software. This is a powerful tool for selecting the regions automatically. Some parameters must be adjusted before or during the selection process in this tool, as seen in Figure 2. The most important parameter in this tool is the Value Tolerance which is used to expand the selection to all image points as long as the difference between the pixel value of the point clicked and the image point is less than the Value Tolerance. This parameter is adjusted to be able to select the scar completely in this study. In addition, the connectedness was set to "8-connected" and "include holes" was checked. Other parameters were used as default.

The photograph must be calibrated to obtain a result in SI units in the measurements and calculations. For calibration, the scale's photograph is taken together with the victim's photograph and by using the line tool, the value of a selected length of the pixels can be obtained in SI unit by using this scale. This value is then entered into the Set Scale tool of the ImageJ for calibration.

To simplify the analysis process, a menu that includes only the required tools was designed using JAVA language as seen in Figure 3.

\section{Results and Discussions}

Some example photographs of the facial scars are represented in Figure 4. These photographs were taken a minimum of 6 months after the trauma when the healing is completed. It is easily seen that these scars are visible by the naked eye, and

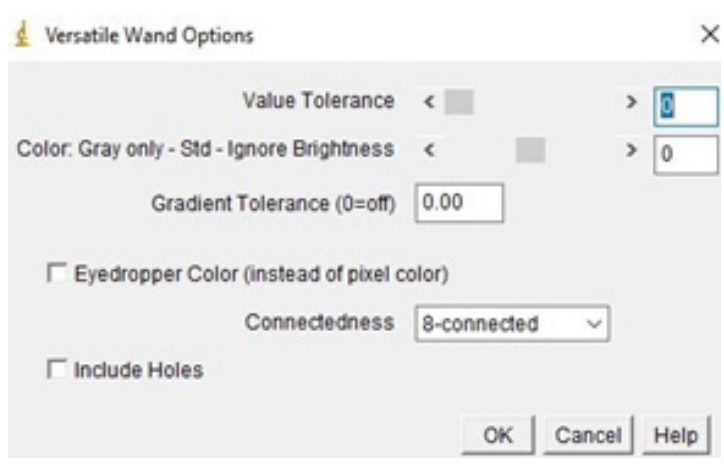

Figure 2: The parameters of the Versatile Wand Tool plugin used in ImageJ. 
they can be assumed as permanent and conspicuous facial scars.

The photographs were taken under different lighting conditions to obtain clearer photographs, and some examples are shown in Figure 5. It is seen that when the polarized filter and white light illumination are used, the visibility of the scar

\begin{tabular}{|c|c|}
\hline d YuBITA & - \\
\hline & Open \\
\hline & Save \\
\hline Zoom & Open a Duplicate \\
\hline \multicolumn{2}{|c|}{ Region of Interest } \\
\hline \multicolumn{2}{|c|}{ Versatile Wand Tool } \\
\hline \multicolumn{2}{|c|}{ Versatile Wand Tool Options } \\
\hline Line Tool & Plot Profile \\
\hline & te Report \\
\hline
\end{tabular}

Figure 3: The menu is written in JAVA language, which includes only the required tools for the analysis
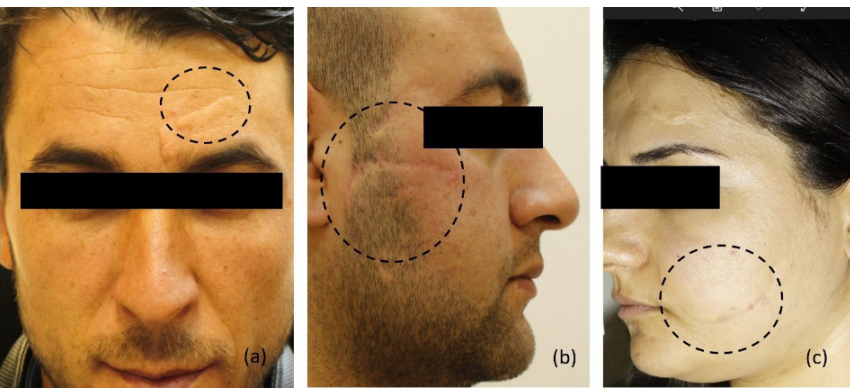

Figure 4: Some examples photographs which show facial scars. is better, as shown in Figure 5(a). When other colors are used for lighting, information in the image is lost partly.

The quantitative analysis of these scars is crucial for preparing an objective forensic medical report. The first step of the analysis is determining the relative color change between the scar region and the nearby intact area on the face. The ImageJ plot profile tool was used to determine the relative color change as mentioned in the Materials and Methods section. Here, the most essential point is selecting the point where the line will be drawn to obtain the relative color change. The criterion of the selection of this point is maximizing the relative color change. This can be performed by moving the line which passes through the scar-healthy region in the "live" mode of the plot profile tool and deciding a line for which the contrast between the intact and the scar regions is maximum. Some example pictures of this procedure are shown in Figure 6 and it is seen that the upper left profile has the maximum contrast between the scar and nearby healthy regions where the color values for the scar are higher than the color values of the healthy part.

After the line decides for plotting the color profile, the relative color change can easily be calculated by taking the ratio of the average color values of the scar and the nearby healthy regions.

The second step of the analysis is the selection and the calculation of the surface area of the scar region as seen in
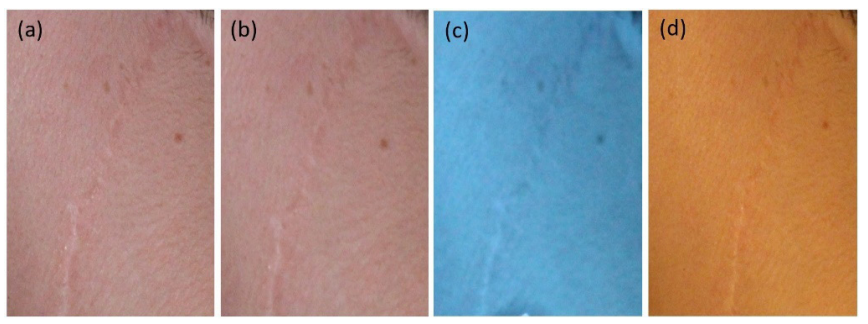

Figure 5: The photographs were taken under different conditions; white light with polarized filter (a), white light (b), blue light (c), and yellow light (d)

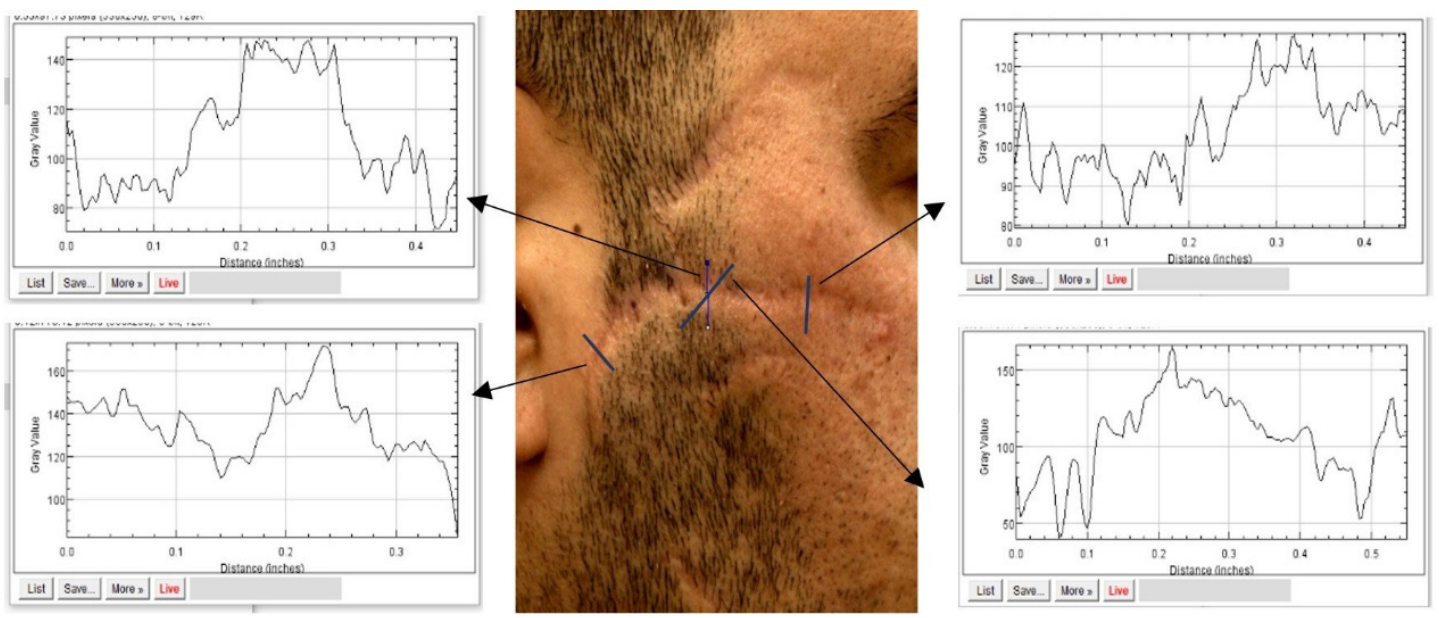

Figure 6: Different colour profiles through the lines drawn at different points of the scar-nearby healthy region. Here, it is seen that the upper left profile has the maximum contrast between the scar region and the intact area 
Figure 7. After selecting the scar region, it must be marked and added as a region of interest. This can be done by using the region of interest (ROI) tool of the ImageJ software. The selected regions can be added to the ROI list by clicking the "Add" button in the ROI manager. Sometimes, the scar region cannot be selected as a single part. Some small regions are selected to cover the scar completely in these cases, and each region is added to the ROI manager list. After adding these selections to the list, they can be combined to obtain one selection covering the whole scar region. The surface area of this combined region can automatically be measured by using the "Measure" tool in the ROI manager.

In quantitative analysis of the photographs, it is expected that most of the deviation will result from the selection of the scar region. Here, the tolerance in the Versatile Wand tool depends on the person who makes this analysis, and the value of the tolerance will change the value of the surface area of the scar region. In Figure 8, the variation of the surface area of the same scar region selected by different people at different times was shown. From this variation, it was concluded that the amount of the deviation in the selection of the scar is about $12 \%$ and it can be assumed that it does not have a considerable effect on the results of the analysis.

Many photographs were analyzed successfully by following the procedures mentioned above. Some example photographs and analyses were given in the following figures.

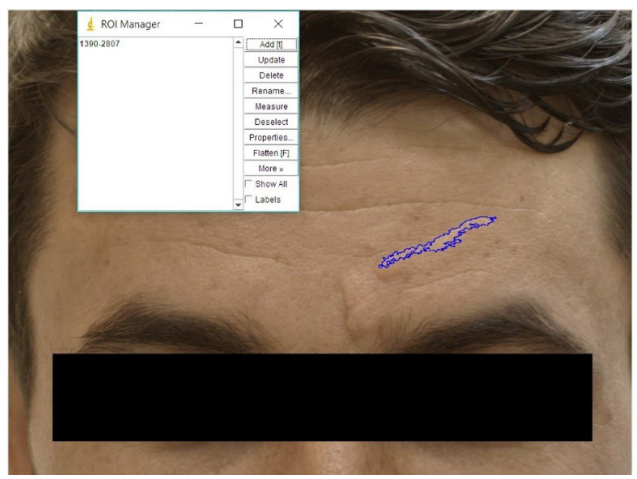

Figure 7: A photograph with a selected scar region and the region of interest (ROI) tool of Image J

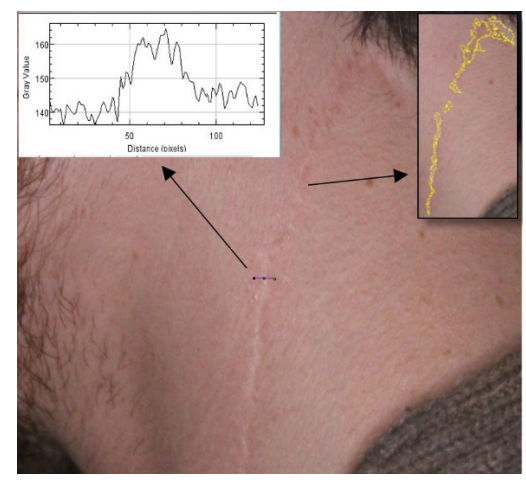

Figure 9: The scar is seen on the neck of the victim. The plot of the colour change through the line and the selection of the scar was given in the inset figures
In Figure 9 the scar region on the neck of the victim was shown. The relative colour change for this scar is about $16 \%$ and the surface area of the selected scar region is $266.9 \mathrm{~mm}^{2}$. In addition to the surface area, the length of the scar was also measured by using polynomial approximation to $116.7 \mathrm{~mm}$. The visibility of the scar region by the naked eye is clear and the decision of the conspicuousness of permanent scar can easily be given for this victim.

In Figure 10, the scar region is seen on the chin of the victim. The relative colour change for this scar was found as $20 \%$, which is higher than the relative change obtained for the victim given in Figure 9 even if the scar region is bigger. This is probably due to the location of the scar. In Figure 10, the skin's surface is covered by the residue beard after shaving, which darkens the color of the intact region. Thus, the relative color change is more pronounced for this victim. The surface area and the length of the selected scar region were measured as $30.1 \mathrm{~mm}^{2}$ and $15.5 \mathrm{~mm}$, respectively.

Another example of the scar which is visible with the naked eye is shown in Figure 11. Here, the visibility of the scar region differs for different parts of the scar. The bottom part of the scar is more pronounced than the upper part. The relative color change analysis of the scar must be performed as the previous analysis and the maximum change must be chosen at the end. In addition, a relatively long beard decreases the visibility of the scar and for correct analysis,

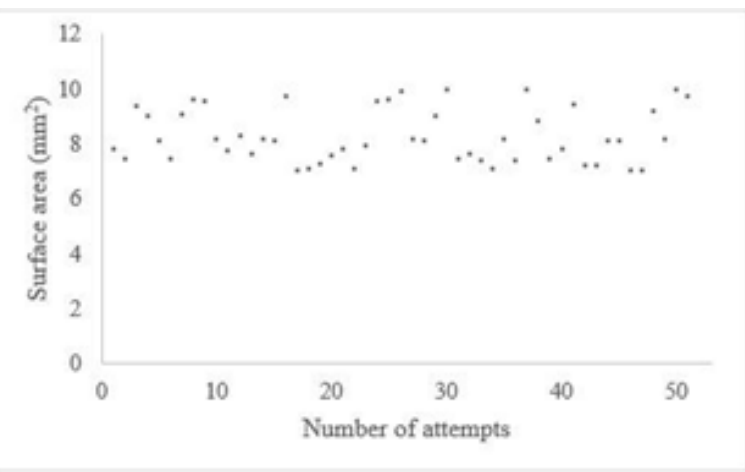

Figure 8: Variation of the surface area of the same scar region depending on the selection of the scar region at different times by different people

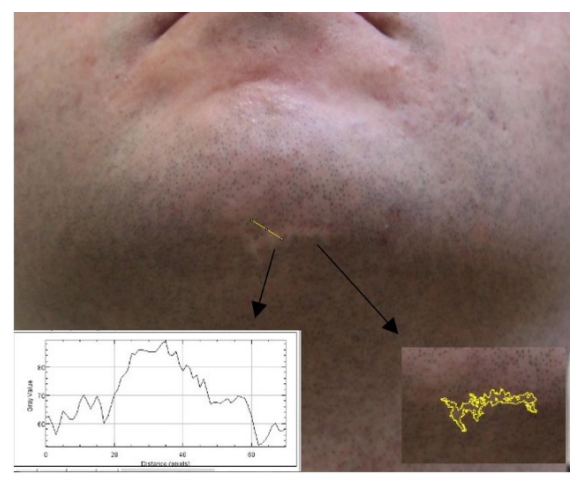

Figure 10: The scar region on the chin of the victim. The plot of the colour change through the line and the selection of the scar was given in the inset figures 
it must be shaved before the analysis. The length of the scar again was calculated by using a polynomial approximation and found to be $104.7 \mathrm{~mm}$. The surface area of the selected scar region was calculated as $272.9 \mathrm{~mm}^{2}$.

The conspicuousness of permanent facial scar can easily be given for the victims given in Figures. 9, 10 and 11 by using just the relative color changes and with the observation by the naked eye. However, the scars are given in the following Figures. 12, 13 and 14 are not visible to the naked eye and the decision of conspicuousness of permanent facial scar is not easy to make. In addition to the relative colour change, other physical properties such as the length and the surface area of the scar region must also be taken into account before the final decision for these types of scars.

In Figure 12, the scar on the upper lip was shown. In the upper inset figure, the plot profile was given and the relative colour change between the scar region and the intact area was measured as $8 \%$. In the bottom inset figure, the selection of the scar region was given and from this selection, the surface area of the scar region was calculated as $17.8 \mathrm{~mm}^{2}$. The visibility of the scar region with the naked eye concerning nearby healthy region is not clear enough so the decision of conspicuousness of permanent facial scar cannot be made easily for this victim.

In Figure 13, a scar on the temple is shown. As seen in this figure, the scar is not clear enough to observe by the naked eye. For this scar, the relative color change was found to be $6 \%$.

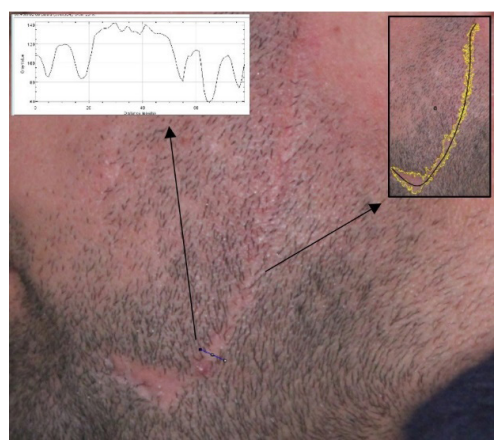

Figure 11: The scar on the cheek of the victim. The plot of the colour change through the line and the selection of the scar was given in the inset figures

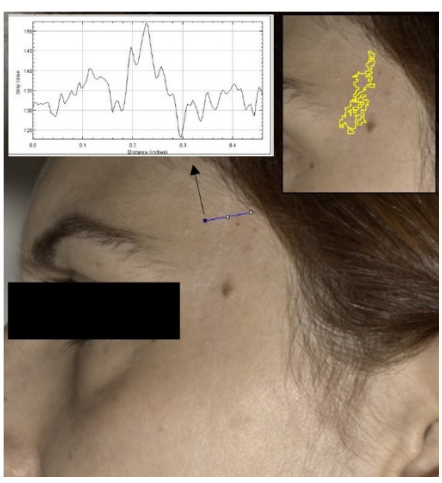

Figure 13: A scar on the temple. The plot of the color change through the line, and the selection of the scar was given in the inset figures
The surface area and the length of the scar were measured as $38.1 \mathrm{~mm}$ and $160.7 \mathrm{~mm}^{2}$.

Another example of these types of scars is given in Figure 14. Here, the scar region is fairly visible by the naked eye again, and the relative color change for this scar was calculated as $9 \%$. The surface area and the length of the scar were measured as $12.1 \mathrm{~mm}$ and $21.2 \mathrm{~mm}^{2}$.

After examining 100 cases in the $2^{\text {nd }}$ Specified Committee of Council of Forensic Medicine, it was concluded that the final decision of conspicuousness of permanent facial scar could easily be given under the observation by physicians' naked eye when the relative color change is bigger than $15 \%$. Whereas, for the victims with a relative color change smaller than $15 \%$, the observation by the naked eye is not enough to give the decision of the conspicuousness of permanent facial scar objectively. For these cases, the results of the quantitative measurements given in this study must be used together with the observation made by the naked eye.

In the photographs given in Figure 15, this situation is seen clearly. Here, the relative color change of the scars for the left and right photographs was calculated as $6 \%$ and $12 \%$, respectively. Although the relative color change is more extensive for the victim in Figure 15(b), the conspicuousness of permanent facial scar decision was made for the victim in Figure 15(a), not for the victim in Figure 15(b) as a result of the observation by the physicians' naked eye. Here only

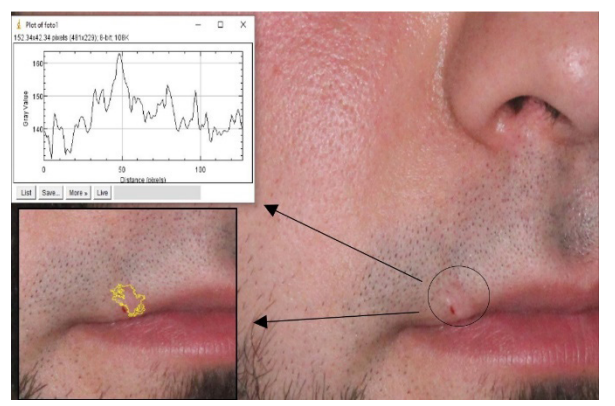

Figure 12: The scar on the upper lip. The plot of the colour change through the line and the selection of the scar was given in the inset figures

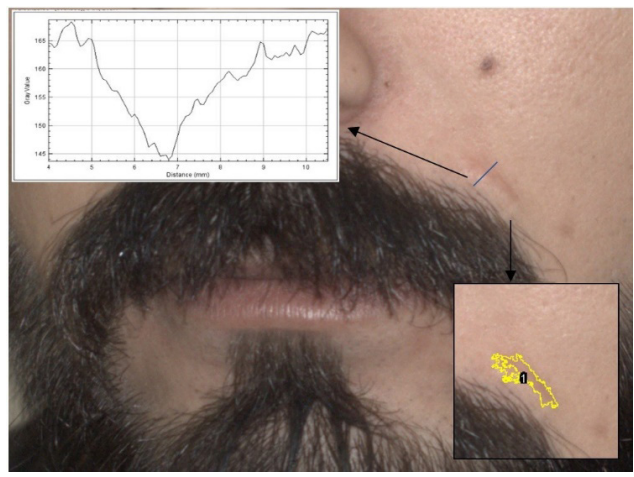

Figure 14: The scar at the borderline of the moustache. The plot of the colour change through the line and the selection of the scar was given in the inset figures 

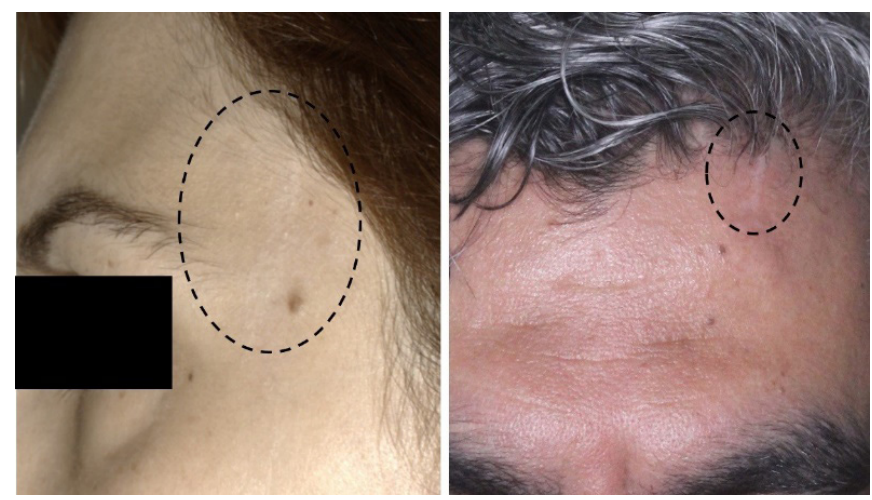

Figure 15: Comparison of two critical facial scars. The relative color change of the scars was calculated as $6 \%$ (left) and $12 \%$ (right)

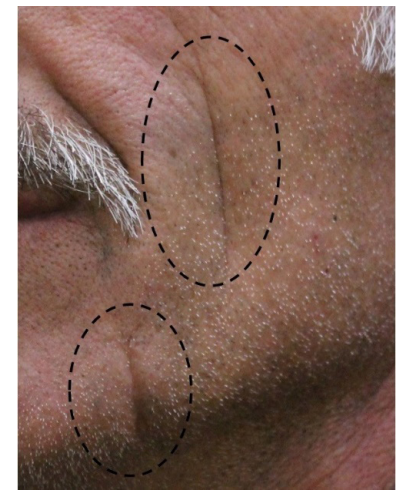

Figure 17: The scar regions on the cheek and chin. These scars are examples of deep scars

one example is given, but it was observed that these kinds of evaluations that appear contradictory mainly were met for the victims who have relative color change below $15 \%$. Therefore, it is recommended that quantitative measurements for these critical cases must be taken into account before the final decision, as in this example.

In Figure 16, the scar on the left ear of the victim was given, and the relative color change, length and the surface area of the scar region were found as $20 \%, 7.2 \mathrm{~mm}$, and 7.3 $\mathrm{mm}^{2}$, respectively. This photograph clearly shows that the image processing-based analysis given in this study can easily be applied to the photographs taken from any body part. It means that the technique proposed in this study can easily be generalized to many forensic applications related to image processing.

In Figure 17, an example of a deep facial scar was given. For these types of scars, 3D imaging of the scar region must be used, and it is more meaningful than the 2D images. Because, by using the $2 \mathrm{D}$ images, only the cross-sectional area can be calculated, which deviates from the real value of these cases. Using the 3D images will be possible to obtain the correct surface area and the volume information of the complete scar.

\section{Conclusion}

It was shown that the image processing-based analysis could be used for quantitative measurement of the physical

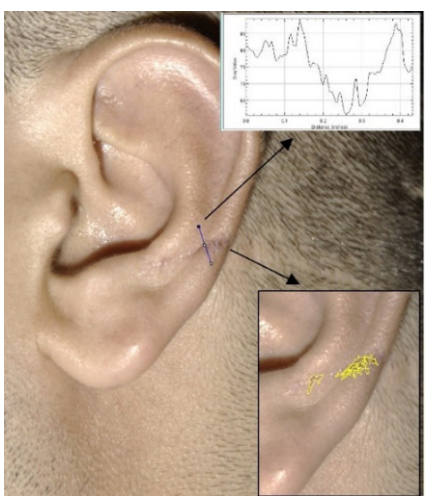

Figure 16: The scar on the ear. The technique proposed in this study can be generalized to any part of the body

properties of the facial scars. The analysis gives very precise results, and these results can be used for the objective decision of the conspicuousness of the permanent facial scar.

Although parameters such as the location of the scar tissue, its dimensions, accompanying age lines, etc. are important, it is considered that the $15 \%$ and/or above relative color difference for a significant part of the cases will be effective alone in deciding for the conspicuousness of permanent facial scar. However, for some exceptional cases, if the physician gives the opposite opinion despite these relatively high color changes, this decision should be explained and supported by some other evidence. One example of this can be the location of facial scars such as surrounded by beard or moustache. It should be highlighted that if the surrounding area of the scar is suntanned; the decision should be postponed until the tanned skin goes back to its normal color.

As contrarily, a decision for conspicuousness of permanent facial scar can be given by physician although the relative colour change of scar is lower than $15 \%$, but this decision should also be based on the quantitative results such as volume, square, length and location of scar or explained by physician's external observation.

Most errors might occur from the selection of the scar region because, during the selection, the operator adjusts the tolerance of the selection, and some fluctuations might probably arise depending on the operator. Therefore, care should be given for some border cases with a little less than $15 \%$ relative color change because, during the selection process, the operator's adjustment to select the value tolerance may affect the results. However, as it was calculated that the deviation result from this part is about $12 \%$, and it does not have a considerable effect on the analysis.

\section{ACKNOWLeDgement}

We would like to thank the director of the Turkish Council of Forensic Medicine for his contribution.

\section{REFERENCES}

1. Saukko P, Knight B. Knight's Forensic Pathology. 3rd ed. London: CRC Press; 2004. 
2. Davis N. Forensic medicine: clinical and pathological aspects. Medico-Legal Journal. 2003;71(1):41-41. Available from: doi. org/10.1258/rsmmlj.71.1.41

3. Vetter F, Pereira RT, Block LL, Zaitter WM, Oliveira RN de, Fernandes MM. Critical analysis of methodologies for valuation of esthetic damage and the forensic application in brazil. RSBO. 2018;14(3):177-185. Available from: doi.org/10.21726/rsbo. v14i3.663

4. McGrouther DA. Facial disfigurement: the last bastion of discrimination. BMJ. 1997;314:991. Available from: doi. org/10.1136/bmj.314.7086.991

5. Enqvist B, Konow L von, Bystedt H. Pre- and perioperative suggestion in maxillofacial surgery: effects on blood loss and recovery. International Journal of Clinical and Experimental Hypnosis.1995;43(3):284-294. Available from: doi.org/10.1080/ 00207149508409971
6. Newell R, Marks I. Phobic nature of social difficulty in facially disfigured people. British Journal of Psychiatry. 2000;176(2): 177-181. Available from: doi.org/10.1192/bjp.176.2.177

7. VanSwearingen J. Facial Rehabilitation: A Neuromuscular Reeducation, Patient-Centered Approach. Facial Plastic Surgery. 2008;24(2):250-259. Available from: doi.org/10.1055/s-2008-1075841.

8. Shepherd JP. Strategies for the study of long-term sequelae of oral and facial injuries. Journal of Oral Maxillofacial Surgery. 1992;50(4):390-399. Available from: doi.org/10.1016/02782391(92)90405-O

9. Ozgenc I. General Principles of Turkish Penalty Code. 9th ed. Ankara: Seckin Press; 2013.

10. ImageJ User Guide. Available from: https://imagej.nih.gov/ij/ docs/guide/

11. Versatile Wand Tool. Available from: https://imagej.nih.gov/ij/ plugins/versatile-wand-tool/index.html 